Research Article

\title{
The Protective Effect of Cyanidin-3-Glucoside on Myocardial Ischemia-Reperfusion Injury through Ferroptosis
}

\author{
Xin Shan, ${ }^{1,2}$ Zhi-Yang Lv $\mathbb{D}^{2}$ Meng-Jiao Yin, ${ }^{1}$ Jing Chen $\mathbb{D}^{2}{ }^{2}$ Jie Wang, $^{2}$ and Qi-Nan Wu $\mathbb{D}^{1}$ \\ ${ }^{1}$ Jiangsu Collaborative Innovation Center of Chinese Medicinal Resources Industrialization, Nanjing University of Chinese Medicine, \\ Nanjing, 210023 Jiangsu, China \\ ${ }^{2}$ Hanlin College, Nanjing University of Chinese Medicine, Taizhou, 225300 Jiangsu, China
}

Correspondence should be addressed to Qi-Nan Wu; qnwyjs@126.com

Received 25 September 2020; Revised 20 January 2021; Accepted 25 January 2021; Published 8 February 2021

Academic Editor: H. P. Vasantha Rupasinghe

Copyright ( 2021 Xin Shan et al. This is an open access article distributed under the Creative Commons Attribution License, which permits unrestricted use, distribution, and reproduction in any medium, provided the original work is properly cited.

This study was conducted to estimate the protective effect of Cyanidin-3-glucoside (C3G) on myocardial ischemia-reperfusion (IR) injury and to explore its mechanism. The rats were subjected to left anterior descending ligation and perfusion surgery. In vitro experiments were performed on $\mathrm{H} 9 \mathrm{c} 2$ cells using the oxygen-glucose deprivation/reoxygenation (OGD/R) model. The results showed the administration of C3G reduced the infarction area, mitigated pathological alterations, inhibited ST segment elevation, and attenuated oxidative stress and ferroptosis-related protein expression. C3G also suppressed the expressions of USP19, Beclin1, NCOA4, and LC3II/LC3I. In addition, treatment with C3G relieved oxidative stress, downregulated LC3II/LC3I, reduced autophagosome number, downregulated TfR1 expression, and upregulated the expressions of FTH1 and GPX4 in OGD/R-induced H9c2 cells. C3G could inhibit the protein levels of USP19 and LC3II. C3G promoted K11-linked ubiquitination of Beclin1. Further evidence that C3G reduced ferroptosis and ameliorated myocardial $\mathrm{I} / \mathrm{R}$ injury was demonstrated with the ferroptosis promoter RSL3. Taken together, C3G could be a potential agent to protect myocardium from myocardial I/R injury.

\section{Introduction}

Cardiovascular disease is a major health problem in industrialised and developing countries, and the high morbidity and mortality have brought heavy healthy and economic burden on society. Cardiac ischemia causes heart tissue damage and myocardial cell necrosis. Pharmacological treatments and early reperfusion therapies can ameliorate the damage; however, these therapies can also cause irreversible myocardial ischemia/reperfusion (I/R) injury, such as deterioration of cardiac function and arrhythmia [1]. Therefore, there is an urgent need to explore effective treatments to reduce myocardial I/R injury.

Ferroptosis is a programmed cell death mediator dependent on iron and ROS [2]. Ferroptosis has been identified in numerous disorders, including cancer, neurodegenerative diseases, and cardiovascular diseases. As a programmed cell death, ferroptosis induces various lipid hydroperoxide- related diseases, including myocardial I/R lesions [3]. Except for necrosis and ferroptosis, autophagy has been suggested to be an important regulator of ischemic/reperfusion dysfunction. Autophagy is an adaptive response to multiple stimuli through the decomposition of harmful proteins and damaged organelles. In myocardial I/R injury, mild autophagy can maintain the intracellular environment and improve the cell survival, but excessive autophagy can cause increased levels of intracellular oxidative stress $[4,5]$. The relationship between autophagy and ferroptosis in cardiac ischemiareperfusion has been limited reported.

Ubiquitination modification governs a number of cellular functions and signaling. Deubiquitinating enzymes (DUBs) reverse ubiquitination by cleaving ubiquitin from the substrate. Ubiquitin specific peptidase (USP) family is one of the DUB subfamilies, among which USP19 is a $150 \mathrm{kDa}$ tail-anchored ubiquitin-specific protease. USP19 participates in the regulation of diverse diseases, such as obesity [5], 
cancer [6], and muscle waiting [7], and proved to be highly related to autophagy. However, whether USP19 can control myocardial I/R injury remains unclear.

Cyanidin-3-glucoside (C3G) is a member of the anthocyanin family which belongs to a subgroup of flavonoids. It is widely distributed in purple or red vegetables and fruits. Recent studies have demonstrated that C3G has anti-inflammatory, antioxidant, hepatoprotective, and cardioprotective effects $[8,9]$. Škèmienè et al. proposed that anthocyanins protected the heart from ischemia/reperfusion-induced apoptosis and necrosis in rats [10]. C3G relieved the doxorubicin-induced cardiotoxicity in mice [11]. C3G also prevented cardiac hypertrophy and diastolic heart dysfunction [12]. Nevertheless, the effect of C3G on myocardial I/R injury has not been fully understood. The present study was to evaluate the pharmacological effect of $\mathrm{C} 3 \mathrm{G}$ on myocardial $\mathrm{I} / \mathrm{R}$-induced rats and investigate its potential mechanism.

\section{Experimental Methods}

2.1. Reagent. Diltiazem (DIL) was purchased from Shanghai Xinyi Wanxiang Pharmaceutical Co., Ltd. (Shanghai, China). Cyanidin-3-glucoside (C3G, \#C832095, with the purity $\geq 98 \%$ ) was obtained from Macklin (Shanghai, China). The anti-USP19 (ab167059), anti-Beclin1 (ab210498), antiTransferrin receptor 1 (TfR1, ab1086), anti-NCOA4 (ab86707), and anti-Ubiquitin (linkage-specific K11) antibodies were provided by Abcam (Cambridge, UK). Anti-ferritin heavy chain (FTH1, \#3998) and anti-LC3I/II (\#12741) antibodies were supplied from Cell Signaling Technology (Boston, USA). Anti-GAPDH antibody, Goat Anti-Mouse IgG $(\mathrm{H}+\mathrm{L})$ HRP second antibody, and Goat Anti-Rabbit IgG $(\mathrm{H}+\mathrm{L}) \mathrm{HRP}$ second antibody were produced by Affinity (Cincinnati, USA).

2.2. Animals and Experimental Protocols. Adult male Sprague Dawley (SD) rats weighing 260-280 g were purchased from Qinglongshan Animal Farm (Nanjing, China). The rats were housed under a $12 \mathrm{~h}$ light/dark cycle at $23 \pm 2^{\circ} \mathrm{C}$ with $40 \%$ $60 \%$ humidity, and they had free access to standard food and water. All animal experimental procedures were approved by the Nanjing University of Traditional Chinese Medicine (Ethical number: No. 201910A246).

After a week of adaptation, the rats were randomly assigned into five groups $(n=8)$ : (1) sham group, rats receiving saline gavage and sham surgery were used as control group; (2) I/R model group, rats receiving saline gavage and left anterior descending (LAD) ligation surgery were used as the model group; (3) C3G-10 group, I/R model plus intraperitoneal injection of $10 \mathrm{mg} / \mathrm{kg} \mathrm{C3G}$; (4) C3G-20 group, I/R model plus intraperitoneal injection of $20 \mathrm{mg} / \mathrm{kg} \mathrm{C} 3 \mathrm{G}$; and (5) DIL group, I/R model plus oral administration of $20 \mathrm{mg} / \mathrm{kg}$ diltiazem. C3G and DIL were dissolved in DMSO and then diluted with saline so that the DMSO concentration was less than $0.1 \%(v / v)$. This procedure was continued for 7 days. Rats were anesthetized with $50 \mathrm{mg} / \mathrm{kg}$ sodium pentobarbital, followed by LAD ligation to occlude the coronary artery for $30 \mathrm{~min}$ before ischemia-reperfusion model of the myocardium was established by releasing the sutures for $2 \mathrm{~h}$ [13]. Simultaneously, the rats in the sham group were subjected to an open-chest step but did not continue with the LAD ligation.

A standard limb lead II electrocardiogram (ECG) was monitored continuously during the procedure. Immediately after the rat was sacrificed, the heart tissue was removed for subsequent experiments.

2.3. TTC Staining. 2,3,5-Triphenyl-2H-tetrazolium chloride (TTC) staining was used to measure the area of myocardial infarction. After the heart had been washed three times with precooled saline, the left ventricle was cut transversely into five sections with the same thickness and stained with TTC at $37^{\circ} \mathrm{C}$ for $15 \mathrm{~min}$. After staining, normal areas of the myocardium were stained red, while infarcted areas were left unstained. Infarct rate $(\%)=$ area of infarcted area/total area $\times 100 \%$.

2.4. H\&E Staining. The heart tissues were fixed in $4 \%$ paraformaldehyde, and the samples were then embedded in paraffin, cut into $4 \mu \mathrm{m}$ thick sections and stained using the hematoxylin-eosin staining method according to the protocol. The histopathological changes in these samples were observed under microscope.

2.5. Detection of ROS. The level of ROS was examined by dihydroethidium (DHE) fluorescent probes (D1008, Yuheng, China) in tissues and 2,7-dichlorodihydrofluorescein diacetate (DCFH-DA) fluorescent probes (D1002, Yuheng, China) in cells. For tissues, the slices were incubated with DHE probe at $37^{\circ} \mathrm{C}$ for $1 \mathrm{~h}$. Representative photographs were taken with a fluorescence microscope, and fluorescence intensities were used to indicate ROS levels. For cells, DCFH-DA fluorescent probe solution at a concentration of $10 \mu \mathrm{M}$ was added to the treated cells and incubated in a cell incubator at $37^{\circ} \mathrm{C}$ for $20 \mathrm{~min}$. The fluorescence intensity was measured using a flow cytometer with excitation/emission wavelengths of $488 / 525 \mathrm{~nm}$, and the average fluorescence intensity was quantified.

2.6. Measurement of Superoxide Dismutase (SOD), Malondialdehyde (MDA), and $\mathrm{Fe}^{2+}$. Superoxide Dismutase (SOD) Assay Kit (A001-3-2, Jiancheng, China), Malondialdehyde (MDA) Assay Kit (A003-1-2, Jiancheng, China), and Iron Assay Kit (ab83366, Abcam) commercial kits were used to determine the relative levels of SOD and the relative levels of $\mathrm{MDA}$ and $\mathrm{Fe}^{2+}$, respectively. All procedures were conducted in accordance with the instructions of the kits.

2.7. USP19 Lentivirus Construction and Infection. Lentivirus particles carrying USP19 (Lv-USP19) and its empty vector (Lv-CON) were constructed by Nanjing Biowarrior Co., Ltd. The lentiviral vectors were transfected at a multiplicity of infection (MOI) of 10 into H9c2 cells. $24 \mathrm{~h}$ later, the culture medium was replaced for $48 \mathrm{~h}$. Then, the infected H9c2 cells were screened with puromycin and cultured for further investigation. Besides, the cells transfected by USP19 lentivirus for $72 \mathrm{~h}$ were used for western blot to confirm the transfection efficiency.

2.8. Cell Culture and Grouping. To estimate the effect of $\mathrm{C} 3 \mathrm{G}$ on $\mathrm{H} 9 \mathrm{C} 2$ cells, the oxygen-glucose deprivation/reoxygenation 
(OGD/R) in vitro model was constructed. The H9C2 cells were donated from Nanjing University of Traditional Chinese Medicine University and cultured in DMEM medium containing 10\% fetal bovine serum (FBS) and double antibiotics (penicillin and streptomycin, $100 \mu \mathrm{g} / \mathrm{mL}: 100 \mu \mathrm{g} / \mathrm{mL}$ ) at $37^{\circ} \mathrm{C}$.

$\mathrm{H} 9 \mathrm{c} 2$ cells were treated with $\mathrm{OGD} / \mathrm{R}$ to simulate in vivo I/R conditions. The cells were divided into five groups: control group, OGD/R group, 25-C3G group, 50-C3G group, and $100-\mathrm{C} 3 \mathrm{G}$ group. $\mathrm{H} 9 \mathrm{c} 2$ cells at $5 \times 10^{4}$ cells/well were seeded into six-well plates and incubated normally for $24 \mathrm{~h}$; then, the medium was replaced with low-sugar serum-free DMEM; 25, 50, and $100 \mu \mathrm{M}$ C3G were added to the corresponding $\mathrm{C} 3 \mathrm{G}$ administration groups, and the six-well plates were transferred to an incubator under hypoxic conditions $\left(1 \% \mathrm{O}_{2}, 5 \% \mathrm{CO}_{2}\right.$, and $\left.94 \% \mathrm{~N}_{2}\right)$ for incubation at $37^{\circ} \mathrm{C}$ for $2 \mathrm{~h}$. The cells were incubated normally for another $24 \mathrm{~h}$. Cells in the control group were always cultured under normal conditions.

In experiment 3 , the cells were divided into five groups: control group, OGD/R group, OGD/R + C3G $(100 \mu \mathrm{M})$ group, OGD/R+RSL $(8 \mu \mathrm{M})$ group, and $\mathrm{OGD} / \mathrm{R}+\mathrm{RSL}$ $(8 \mu \mathrm{M})+\mathrm{C} 3 \mathrm{G}(100 \mu \mathrm{M})$ group. $5 \times 10^{4}$ cells/well H9c2 cells were seeded into six-well plates for $24 \mathrm{~h}$. Each group was treated according to the appropriate method and OGD/R procedure as above description. Afterwards, all the cells were harvested for further investigation.

2.9. Detection of Cell Viability. H9c2 cells at $5 \times 10^{3}$ cells/well were seeded into the 96 -well plate for $24 \mathrm{~h}$. The cells were treated with different concentration of C3G $(0,25,50$, $100 \mu \mathrm{M}$ ) or not and stimulated with OGD/R process. After treatment, the supernatant was discarded and coincubated $5 \mathrm{mg} / \mathrm{mL} 3$-(4,5-dimethylthiazol-2-yl)-2,5-diphenyltetrazoliumbromide (MTT) at $37^{\circ} \mathrm{C} .4 \mathrm{~h}$ later, DMSO was added to dissolve the purple formazan crystals. The optical density was determined at $570 \mathrm{~nm}$ by an automated microplate spectrophotometer.

2.10. Transmission Electron Microscope (TEM). The cell samples were immersed in the TEM-specific fixed solution provided by Wuhan Seville Biotechnology Co., Ltd. (Wuhan, China) and then dehydrated in steps of $50 \%, 70 \%, 80 \%$, $90 \%, 95 \%$, and $100 \%$ acetone. After dehydration, the samples were embedded in 618 epoxy resin. Ultrathin sections (approximately $75 \mathrm{~nm}$ ) were then cut with a diamond knife and placed on a copper grid coated with carbon film. The samples were stained by uranium acetate for $15 \mathrm{~min}$, washed, and treated with lead citrate containing sodium hydroxide for $10 \mathrm{~min}$. Finally, a transmission electron microscope was employed to observe the section.

2.11. Western Blot Analysis. The heart tissues and H9c2 cells were collected and homogenized by RIPA. The concentrations of the samples were determined using BCA Protein Assay Kit (BCA1-1KT, Merck, Germany) according to the manufacturer's instructions. Equal amount of protein was separated by SDS-PAGE and transferred onto PVDF membrane. Thereafter, the membrane was blocked by $5 \%$ skim milk at room temperature for $1 \mathrm{~h}$ and incubated with the primary antibody at $4^{\circ} \mathrm{C}$ overnight. After washing, the sample was incubated with horseradish peroxidase-conjugated IgG antibody at room temperature for $1.5 \mathrm{~h}$. The blots were detected using ECL chemiluminescent system (Affinity, Cincinnati, USA) and fluorescent imaging system (ChampChemi, Beijing, China).

2.12. Coimmunoprecipitation (Co-IP). After transfection, drug treatment, and OGD/R procedure, the cells were lysed with lysis buffer. The lysates were incubated with antiBeclin1 antibody or anti-IgG antibody. The samples were precipitated with $50 \%$ protein $\mathrm{A} / \mathrm{G}$ agarose bead solution and slightly shaked overnight at $4^{\circ} \mathrm{C}$. Afterwards, the bound proteins were incubated with anti-K11-Ub antibody for western blot analysis as described above.

2.13. Statistical Analysis. The results were presented as mean \pm SEM. Statistical significance was evaluated using one-way ANOVA with Tukey multiple comparison test. A value of $p<0.05$ was regarded as statistically significant.

\section{Results}

3.1. Effect of C3G on I/R-Induced Myocardial IschemiaReperfusion. The infarct area of the ischemia heart was estimated using TTC staining. As shown in Figures 1(a) and 1(b), I/R stimulation indeed increased the infarct region compared with the sham group, while the administration with $\mathrm{C} 3 \mathrm{G}$ notably reduced myocardial ischemiareperfusion-induced infarction. Besides, the I/R stimulation led to the elevation of ST segment and variation of $\mathrm{T}$ wave. The C3G-20 and DIL groups conducted to the notable downregulation of ST segments, which were more efficient than that in the C3G-10 group (Figure 1(c)). The pathological alteration was evaluated through $\mathrm{H} \& \mathrm{E}$ staining. As presented in Figure 1(d), the clear myocardial structure, arranged fiber tissue, and scarce edema nor inflammatory cell infiltration were observed in the sham group. Whereas exposure to $\mathrm{I} / \mathrm{R}$ contributed to the damaged myocardial structure, disarranged fibers, decreased myocardial cells, nuclear shrinkage, and inflammatory cell infiltration. The treatment with C3G and DIL evidently attenuated the histopathological changes as compared with the I/R group. The above results demonstrated that myocardial ischemiareperfusion causes structural and functional injury to the heart and that $\mathrm{C} 3 \mathrm{G}$ treatment could ameliorate the myocardial I/R injury.

3.2. Effect of C3G on Oxidative Stress Level and $\mathrm{Fe}^{2+}$ Content in Rats with Myocardial I/R. As shown in Figure 2(a), the elevated ROS level in the I/R group was significantly increased compared to the control group and then decreased by the treatment of C3G $(p<0.05)$. SOD and MDA decreased and increased in the I/R group, respectively, but returned towards normal levels in the $\mathrm{C} 3 \mathrm{G}$ group (Figures 2(b) and 2(c)). These results suggest that myocardial I/R modeling increases the level of oxidative stress in the heart tissue, while $\mathrm{C} 3 \mathrm{G}$ decreases this elevation. In addition, we also examined the contents of $\mathrm{Fe}^{2+}$ in the tissues and 


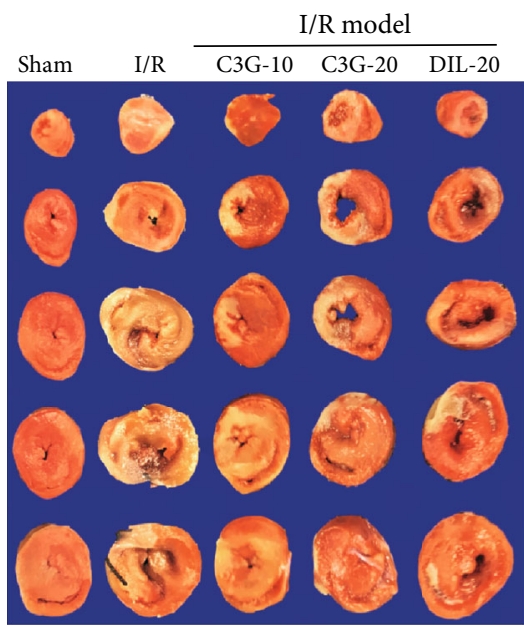

(a)

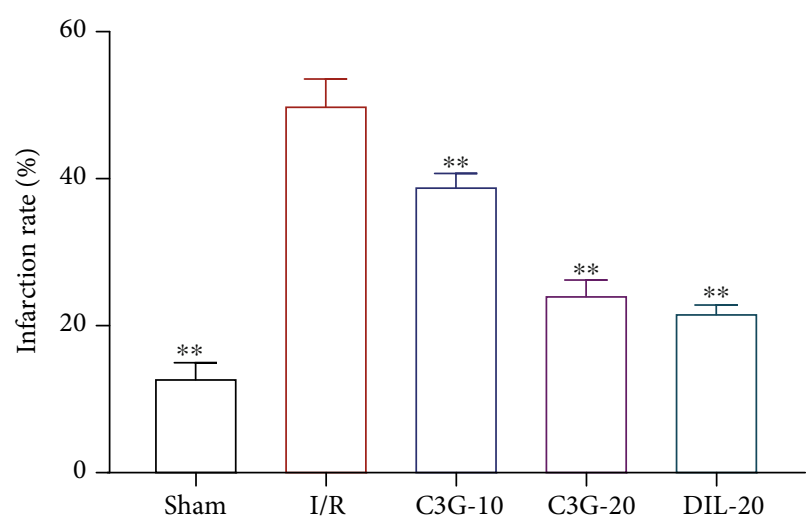

(b)

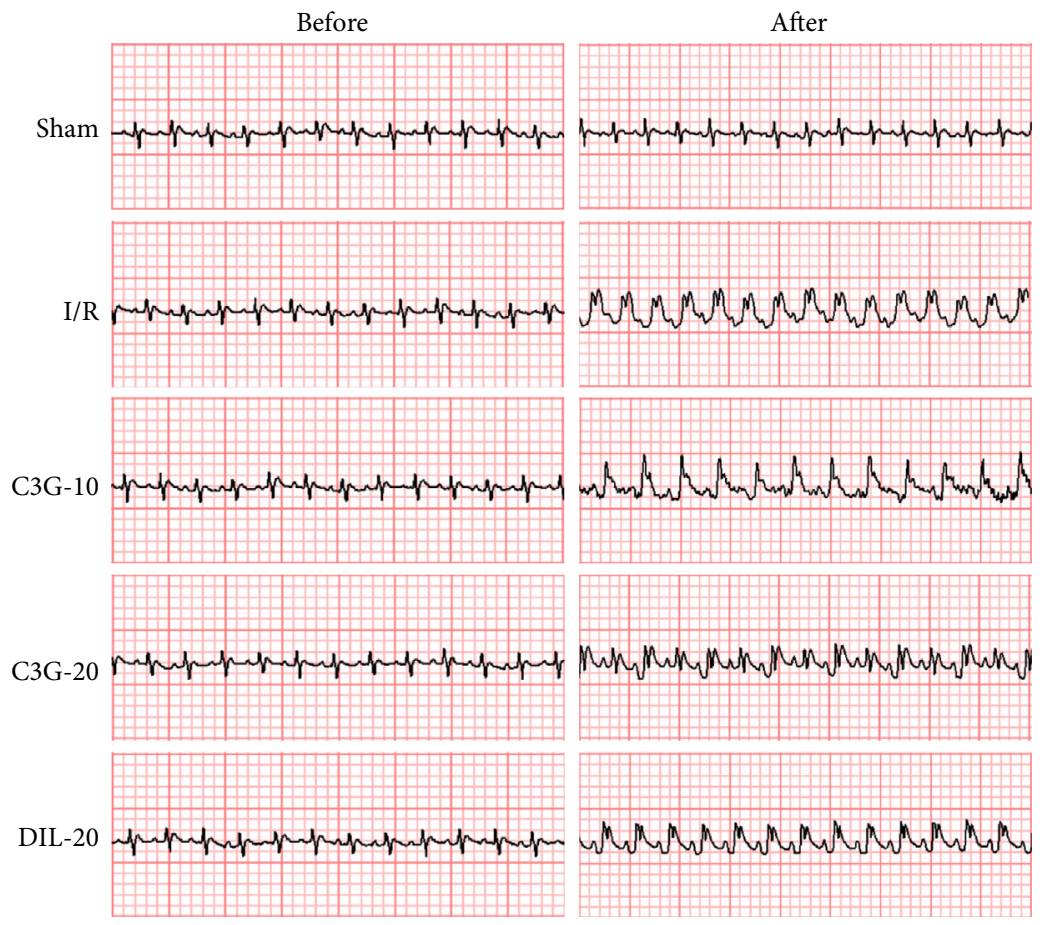

(c)
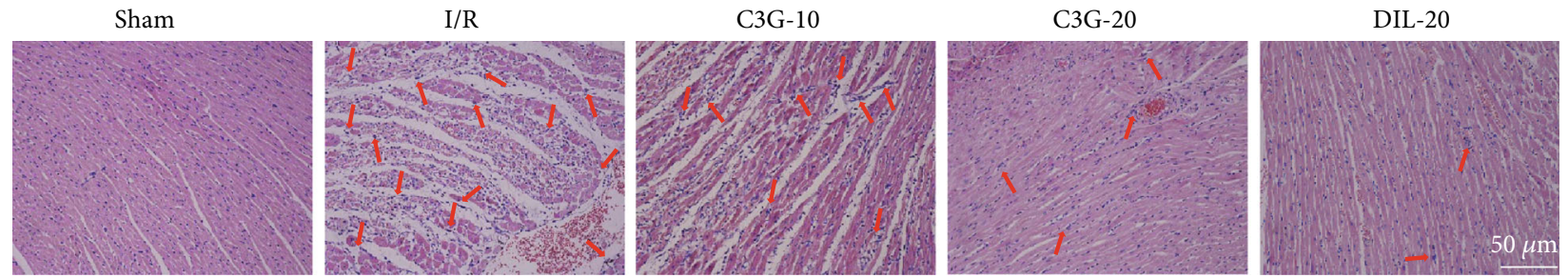

(d)

FIGURE 1: Effect of C3G on I/R-induced myocardial ischemia-reperfusion: (a) the TTC staining; (b) the ratio of infarction rate; (c) representative electrocardiograms before and after modeling; (d) the representative pictures of $\mathrm{H} \& \mathrm{E}$ staining. ${ }^{*} p<0.05,{ }^{* *} p<0.01$ compared with the I/R group.

found that the trend in the changes in $\mathrm{Fe}^{2+}$ contents was consistent with the trend in oxidative stress levels (Figure 2(d)).
3.3. The Effect of C3G on USP19/Beclin1-Mediated Ferroptosis in Heart Tissues of I/R-Induced Myocardial Infarction. To further investigate the potential mechanism of C3G on I/R- 

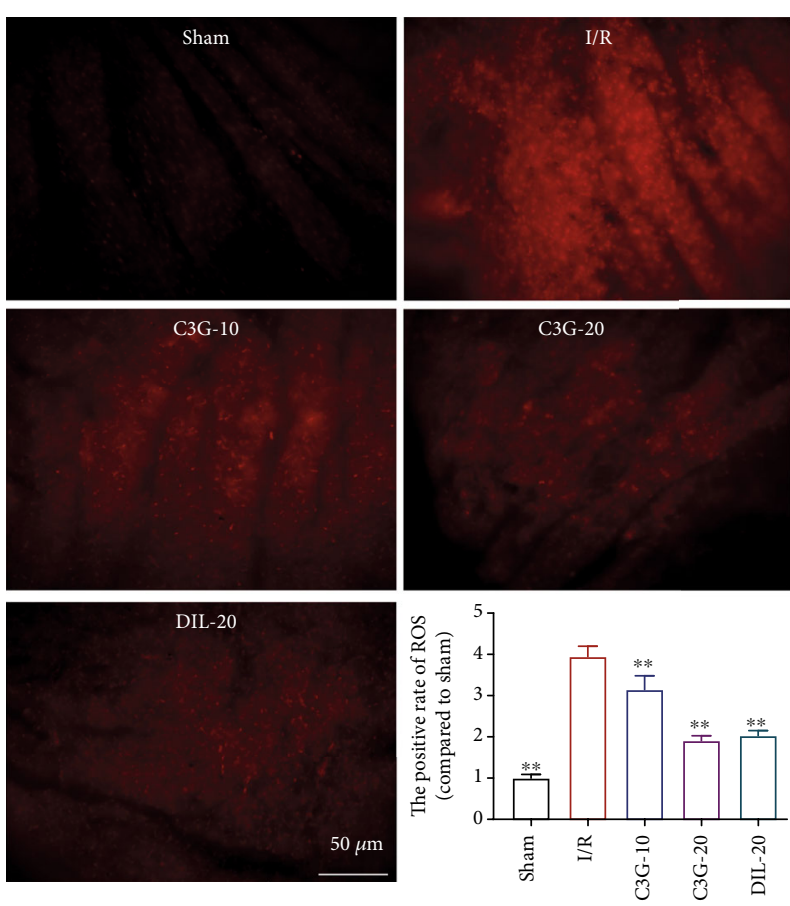

(a)

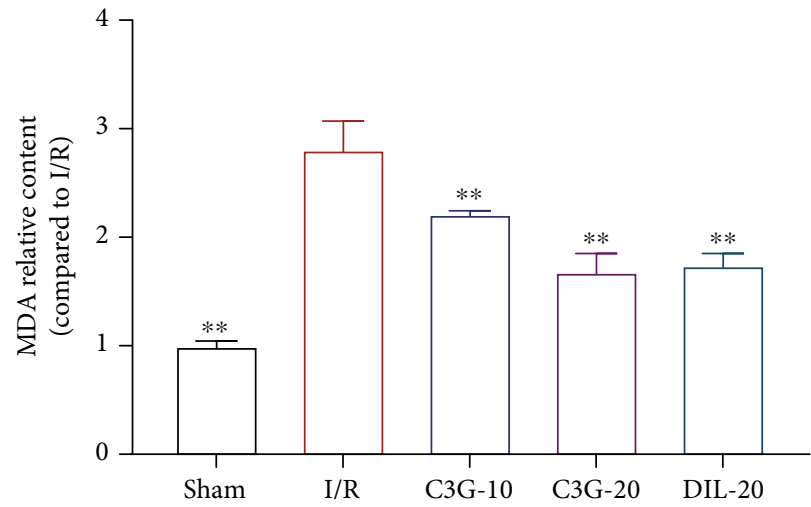

(c)

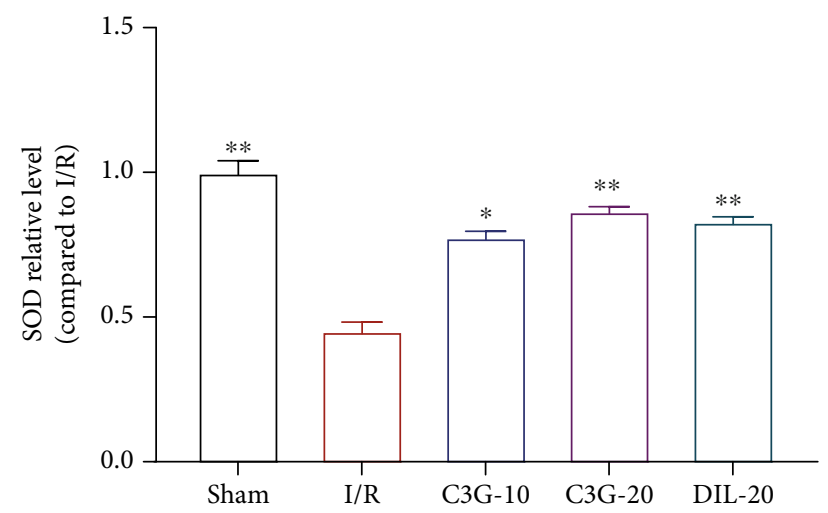

(b)

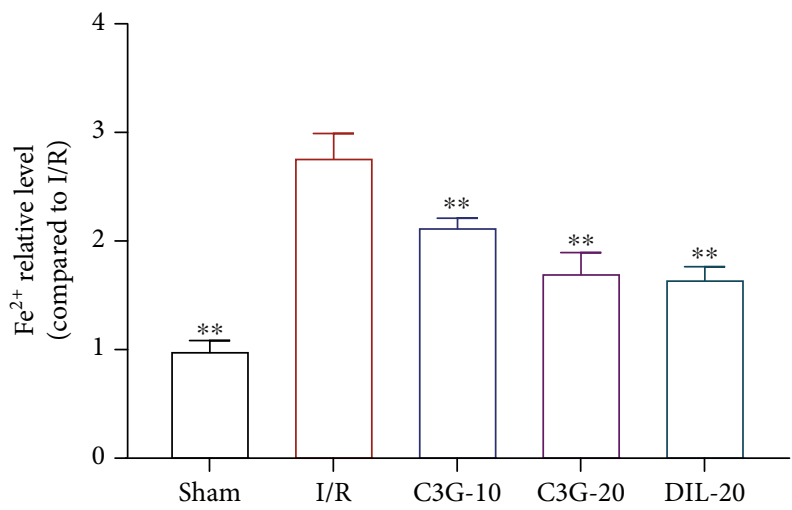

(d)

FIGURE 2: The effect of C3G on oxidative stress level and $\mathrm{Fe}^{2+}$ content in rats with myocardial I/R. (a) The level of ROS was analyzed by immunofluorescence and ratio of ROS positive cells/total cells. (b-d) The levels of SOD, MDA, and $\mathrm{Fe}^{2+}$ in heart tissues of different group. ${ }^{*} p<0.05,{ }^{* *} p<0.01$ compared with the I/R group.

induced cardiac infarction, the expressions of USP19/Beclin1-mediated ferroptosis pathway-related proteins, including USP19, Beclin1, NCOA4, LC3II/LC3I, TfR1, and FTH1 were detected. As illustrated in Figures 3(a) and $3(\mathrm{~b})$, the I/R procedure remarkably increased the protein levels of USP19, Beclin1, NCOA4, and LC3II/LC3I. On the contrary, protein levels of USP19 and Beclin1 were significantly decreased in the C3G-20 and DIL groups, with stronger therapeutic effect than those in the C3G-10 group. The C3G-20 and C3G-10 groups were effectively in reducing the augments of NCOA4 and LC3II/LC3I compared to those of I/R group. C3G treatment also clearly downregulated the protein expression of TfR1 and promoted the expression of FTH1 in I/R-induced cardiac tissues compared to the I/R group. Next, whether C3G influenced the K11ubiqutination was detected by Co-IP. K11-ubiquitination was observed in the sham group; nonetheless, the I/R stimulation evidently blocked the combination between K11ubiquitin and Beclin1, indicating that I/R-induced myocardial ischemia might be associated with deubiquitination. The administration of $\mathrm{C} 3 \mathrm{G}$ could restore the ubiquitination of Beclin1 (Figures 3(c) and 3(d)). The data implied that the protective effect of C3G against I/R-induced myocardial ischemia may be relevant for USP19/Beclin1-dependent ferroptosis.

3.4. The Effect of $\mathrm{C} 3 \mathrm{G}$ on Oxidative Stress Level and $\mathrm{Fe}^{2+}$ Content in $\mathrm{H} 9 \mathrm{c} 2$ Cells. OGD/R modeling caused a distinct increase in number of $\mathrm{DCF}^{+}$cell, indicating an increase in ROS. Nevertheless, the treatment with C3G apparently reduced ROS level compared to that of the OGD/R group (Figure 4(a)). The exposure to OGD/R process dramatically 

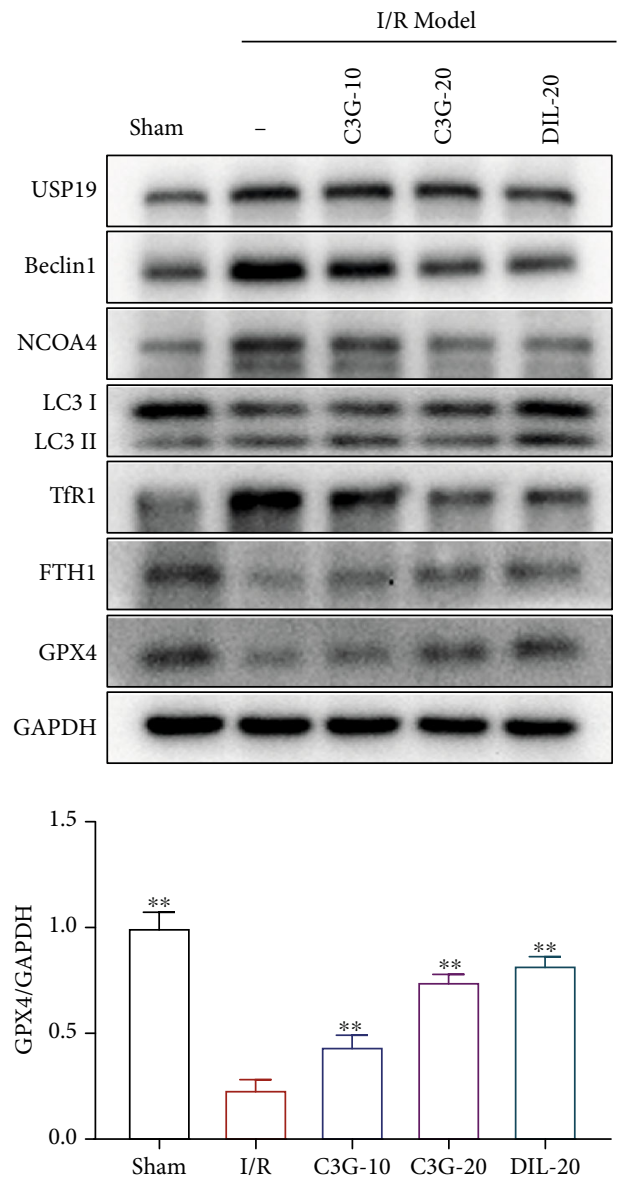

(a)

Figure 3: Continued. 

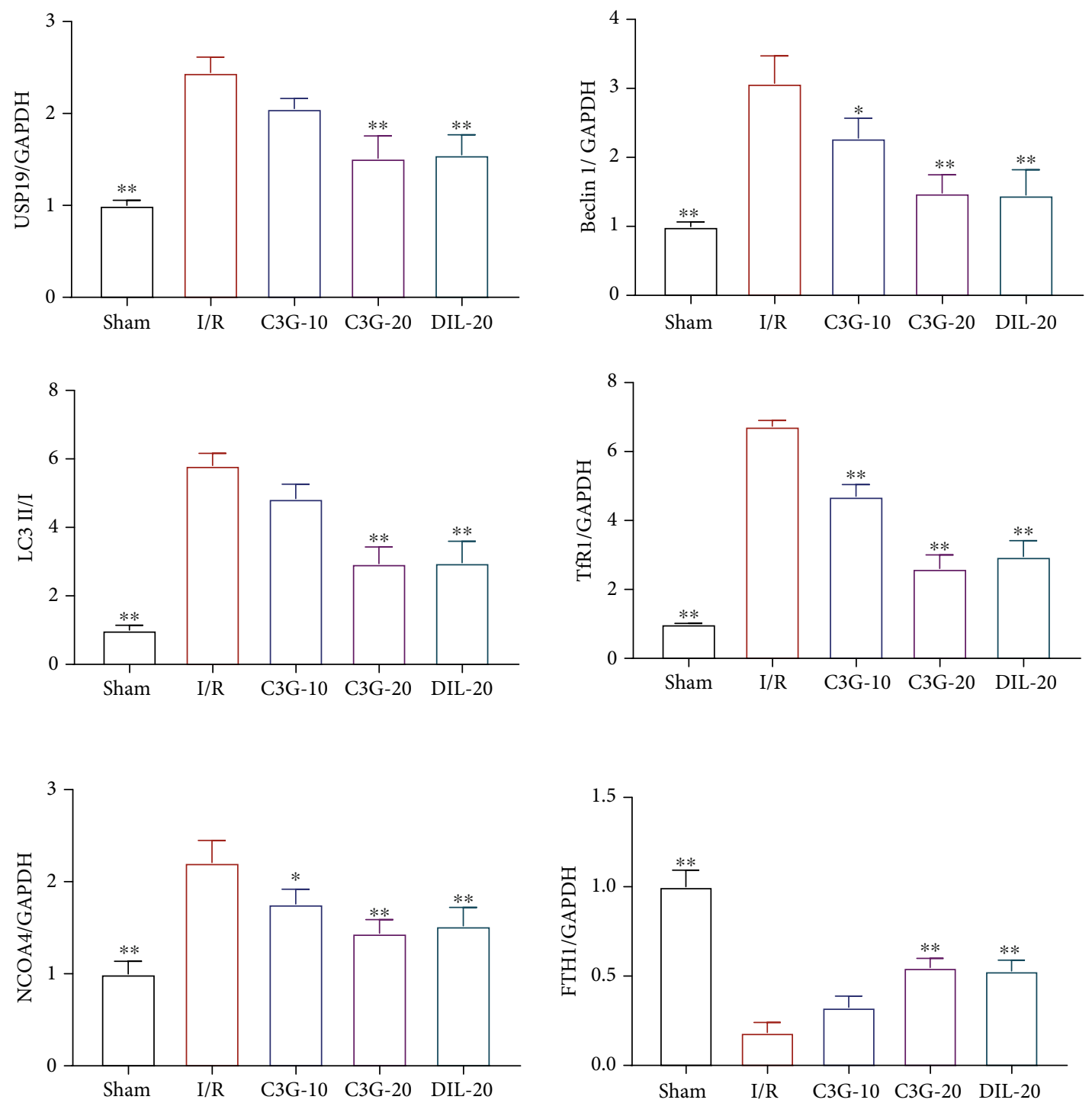

(b)

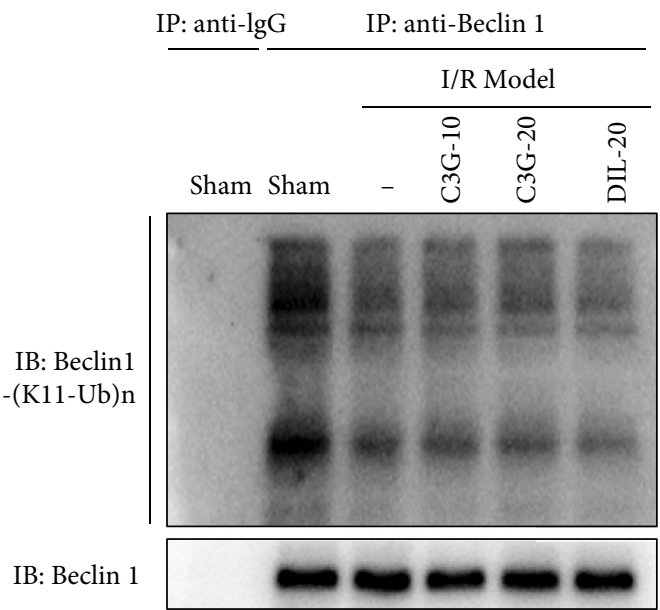

(c)

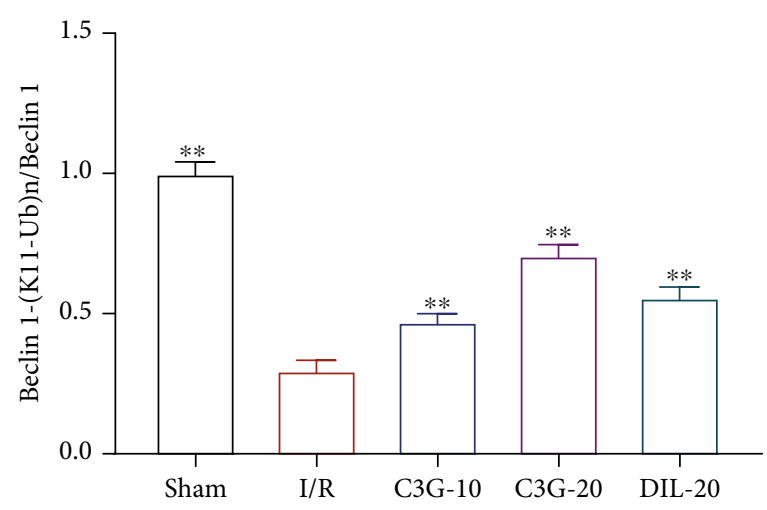

(d)

FIGURE 3: The effect of C3G on USP19/Beclin1-mediated ferroptosis in heart tissues of I/R-induced rats: (a) the effect of C3G on the expressions of USP19, Beclin1, NCOA4, LC3II/LC3I, GPX4, TfR1, and FTH1; (b) the relative levels of USP19, Beclin1, NCOA4, LC3II/LC3I, GPX4, TfR1, and FTH1 versus GAPDH; (c) the effect of C3G on the K11-linked deubiquitination by co-IP; (d) the relative levels of Beclin1-(K11-Ub)/Beclin1. ${ }^{*} p<0.05,{ }^{* *} p<0.01$ compared with the I/R group. 


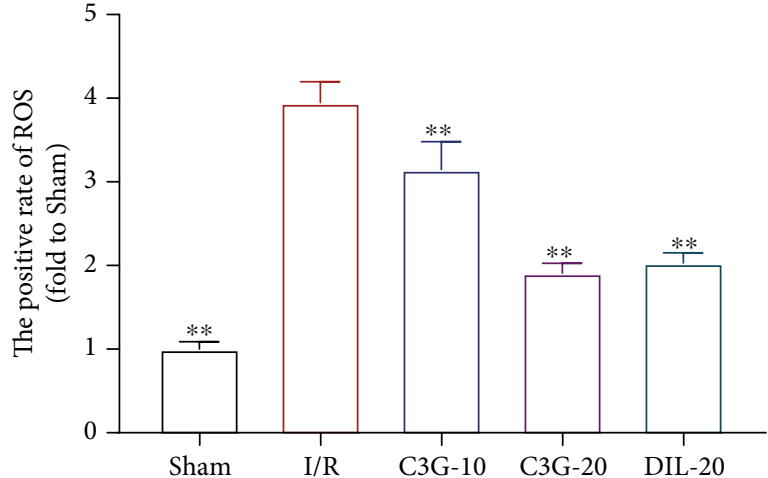

(a)

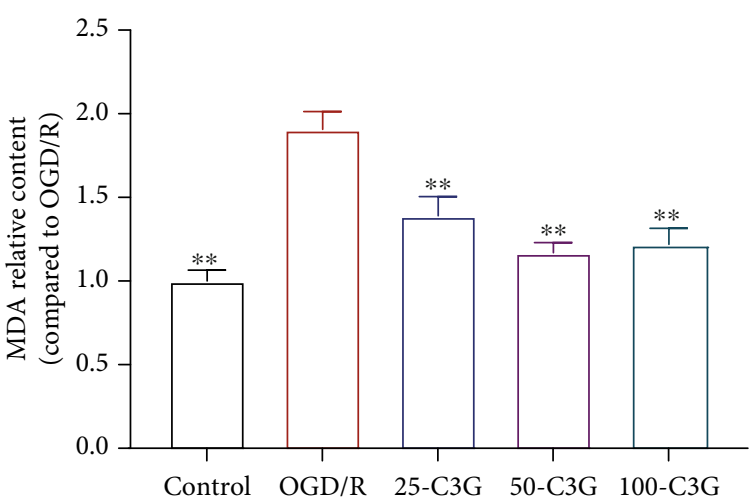

(c)

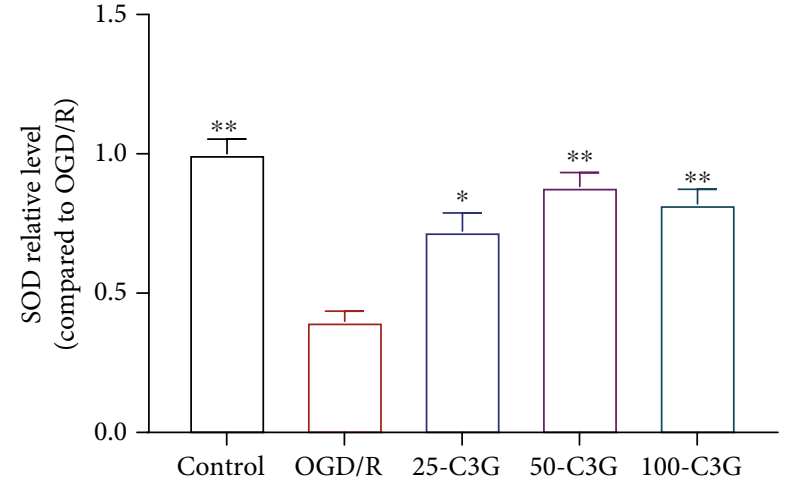

(b)

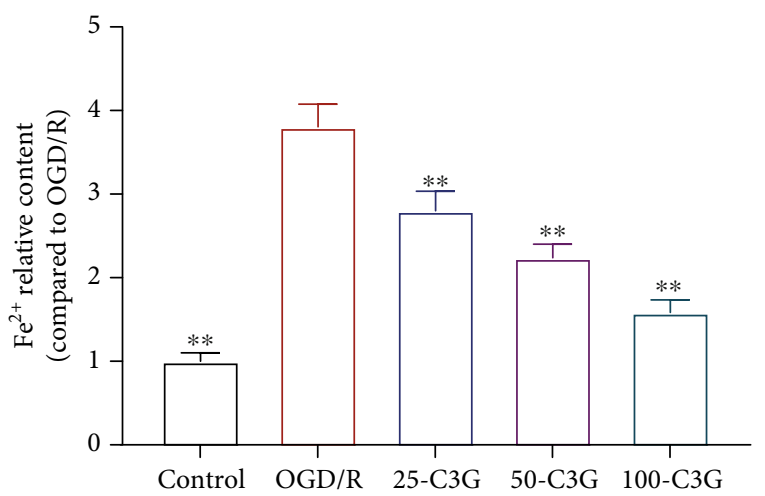

(d)

FIGURE 4: The effect of C3G on oxidative stress level and $\mathrm{Fe}^{2+}$ content in OGD/R-induce H9c2 cells: (a) the relative level of ROS; (b-d) the levels of SOD, MDA, and $\mathrm{Fe}^{2+}$ in cells of different group. ${ }^{*} p<0.05,{ }^{* *} p<0.01$ compared with the I/R group.

promoted of the increase of MDA and $\mathrm{Fe}^{2+}$ and the reduction of SOD activity. In contrast, treatment with C3G $(25 \mu \mathrm{M}$, $50 \mu \mathrm{M}, 100 \mu \mathrm{M})$ conspicuously increased SOD activity and decreased MDA content (Figures 4(b) and 4(c)). In addition, treatment with $\mathrm{C} 3 \mathrm{G}$ reduced $\mathrm{Fe}^{2+}$ content in a concentration-dependent manner (Figure 4(d)). The results proved that $\mathrm{C} 3 \mathrm{G}$ could reduce $\mathrm{OGD} / \mathrm{R}$-induced increases in oxidative stress levels and $\mathrm{Fe}^{2+}$ content in $\mathrm{H} 9 \mathrm{c} 2$ cells.

3.5. The Effect of C3G on Autophagy of OGD/R-Induced H9c2 Cells. The MTT assay confirmed that C3G treatment could increase the viability of cell (Figure 5(a)). The immunofluorescence results showed that $\mathrm{LC} 3 \mathrm{~B}$ expression was upregulated in the $\mathrm{OGD} / \mathrm{R}$ group, which was apparently downregulated in C3G-treated H9c2 cells (Figures 5(b) and 5(c)). Transmission electron microscope was employed to examine the autophagosome in $\mathrm{H} 9 \mathrm{c} 2$ cells. As visualized in Figure 5(d), the number of autophagosomes was increased after OGD/R progression was observed compared with the control group. Whereas, incubation with $\mathrm{C} 3 \mathrm{G}(25 \mu \mathrm{M}$, $50 \mu \mathrm{M}, 100 \mu \mathrm{M})$ led to the reduction of autophagosome number. Our results suggested that the protective effect of C3G on OGD/R-exposed H9c2 cells might be related to the inhibition of autophagy.

3.6. The Effect of C3G on USP19/Beclin1-Mediated Ferroptosis in OGD/R-Induced H9c2 Cells. In vitro, we explored the mechanism of $\mathrm{C} 3 \mathrm{G}$ in OGD/R-induced $\mathrm{H} 9 \mathrm{c} 2$ cells. As expected, the OGD/R model resulted in the upregulation of USP19, Beclin1, NCOA4, and LC3II/LC3I. The treatment with $\mathrm{C} 3 \mathrm{G}(25 \mu \mathrm{M}, 50 \mu \mathrm{M}, 100 \mu \mathrm{M})$ downregulated the expressions of USP19 and LC3II/LC3I in a concentrationdependent manner. The $\mathrm{C} 3 \mathrm{G}$ treatment also reduced the protein levels of Beclin 1 and NCOA4 ferroptosis.

The effects of $\mathrm{C} 3 \mathrm{G}$ on the expressions of TfR 1 and FTH1 were measured. As observed in Figures 6(a) and 6(b), the TfR1 expression was upregulated, and the FTH1 expression was downregulated compared with the control group. The incubation with C3G $(25 \mu \mathrm{M}, 50 \mu \mathrm{M}, 100 \mu \mathrm{M})$ restored the changes in a concentration-dependent manner.

Furthermore, whether $\mathrm{C} 3 \mathrm{G}$ altered the ubiquitination was also assessed in vitro. Ubiquitination of Beclin 1 was reduced in the OGD/R group compared to the control group, indicating that Beclin1 levels were higher in OGD/R than in the control group. However, the C3G $(25 \mu \mathrm{M}, 50 \mu \mathrm{M}$, $100 \mu \mathrm{M})$ incubation promoted the ubiquitination of Beclin1 (Figures 6(c) and 6(d)). Our data proposed that the effect of C3G on OGD/R-induced ferroptosis might be controlled by USP19/Beclin1-induced autophagy.

3.7. The Role of USP19 in the C3G-Mediated Protective Effect and Ferroptosis on OGD/R-Induced H9c2 Cells. In conjunction with the previous results, RSL3, a GPX4 inhibitor, was used to further investigate the protective role of $\mathrm{C} 3 \mathrm{G}$ in 


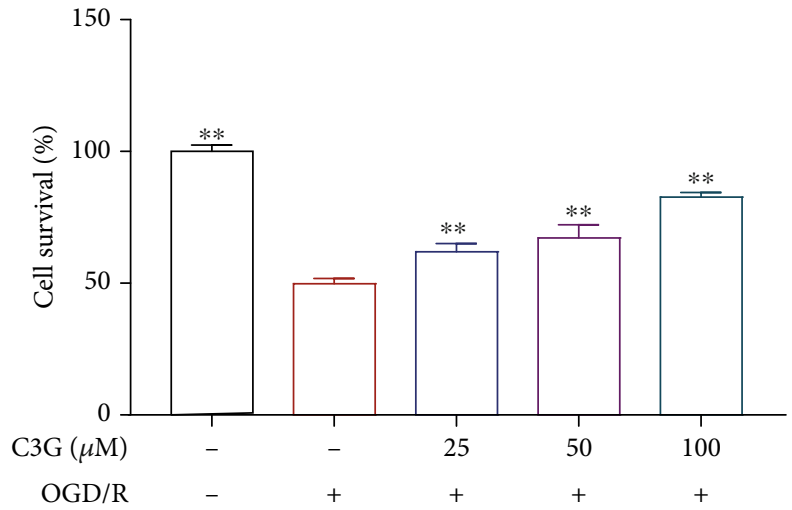

(a)

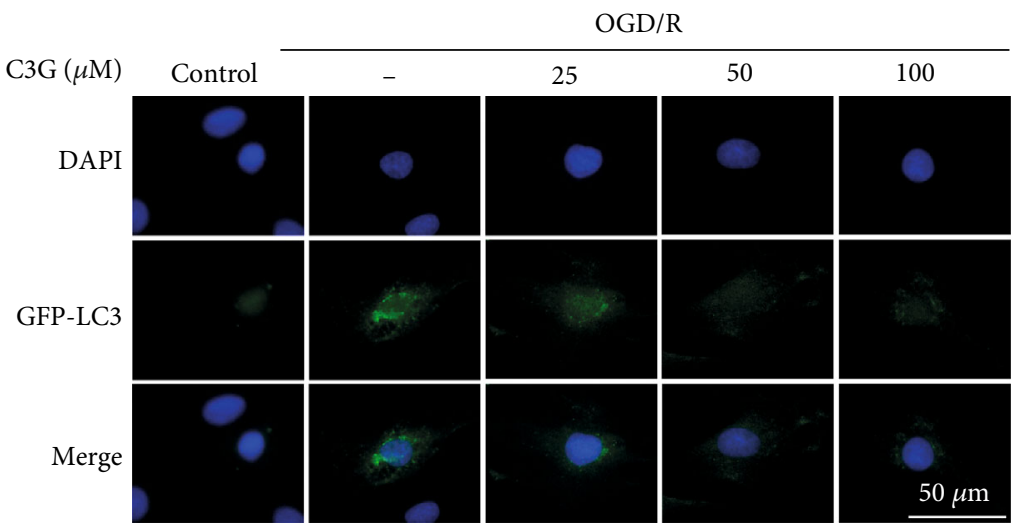

(b)

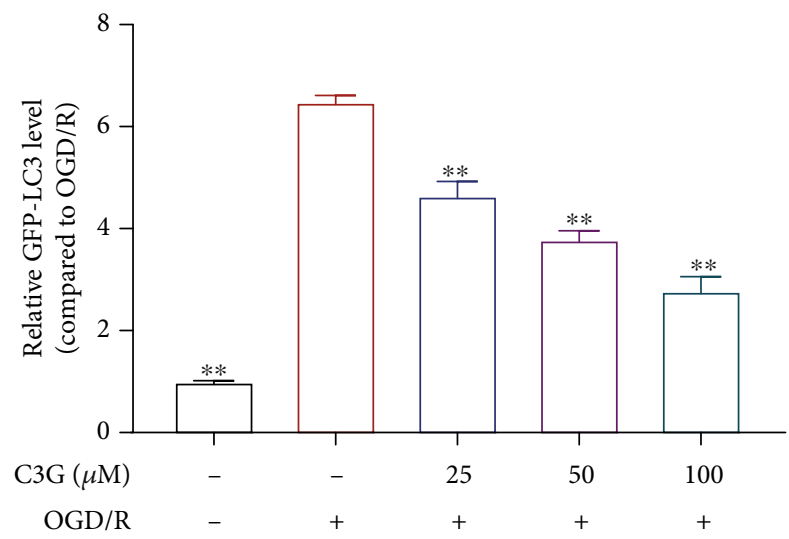

(c)

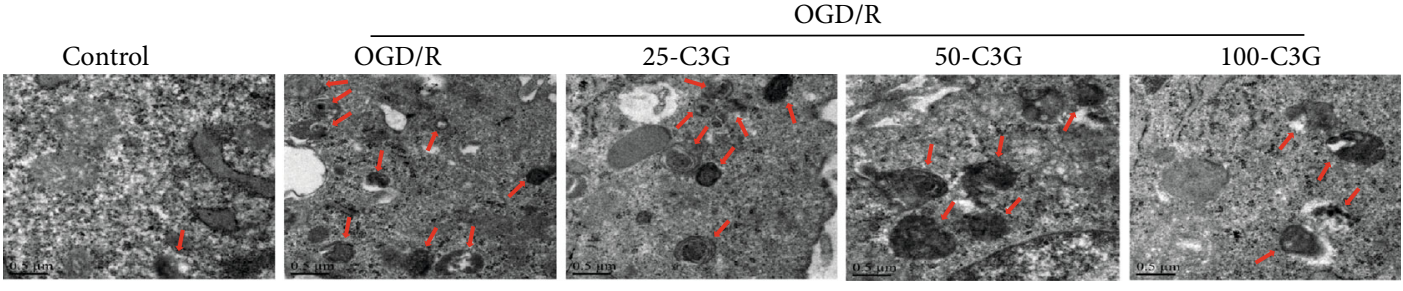

(d)

FIGURE 5: C3G increases cell viability and reduces autophagy. (a) The viability of cell in different group. (b) Representative images of the LC3 expression detected by immunofluorescence. (c) Relative fluorescence intensity of GFP-LC3. (d) The number of autophagosome was observed by transmission electron microscope. ${ }^{*} p<0.05,{ }^{* *} p<0.01$ compared with the OGD/R group. 

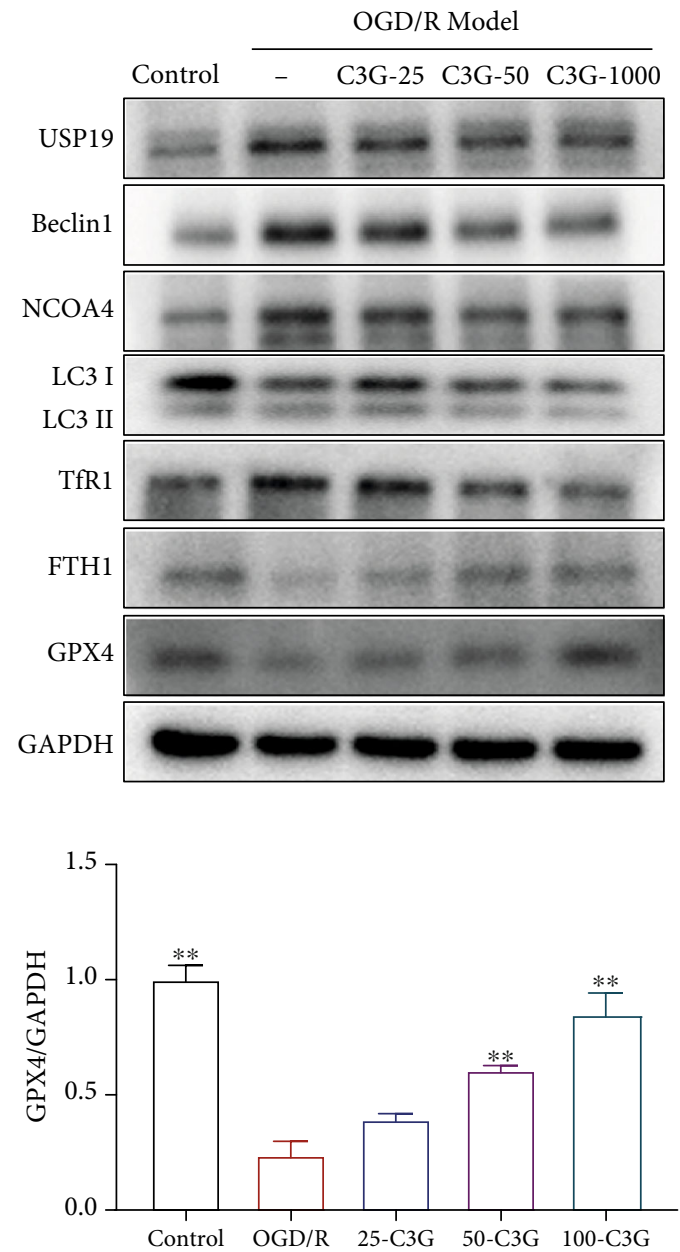

(a)

Figure 6: Continued. 

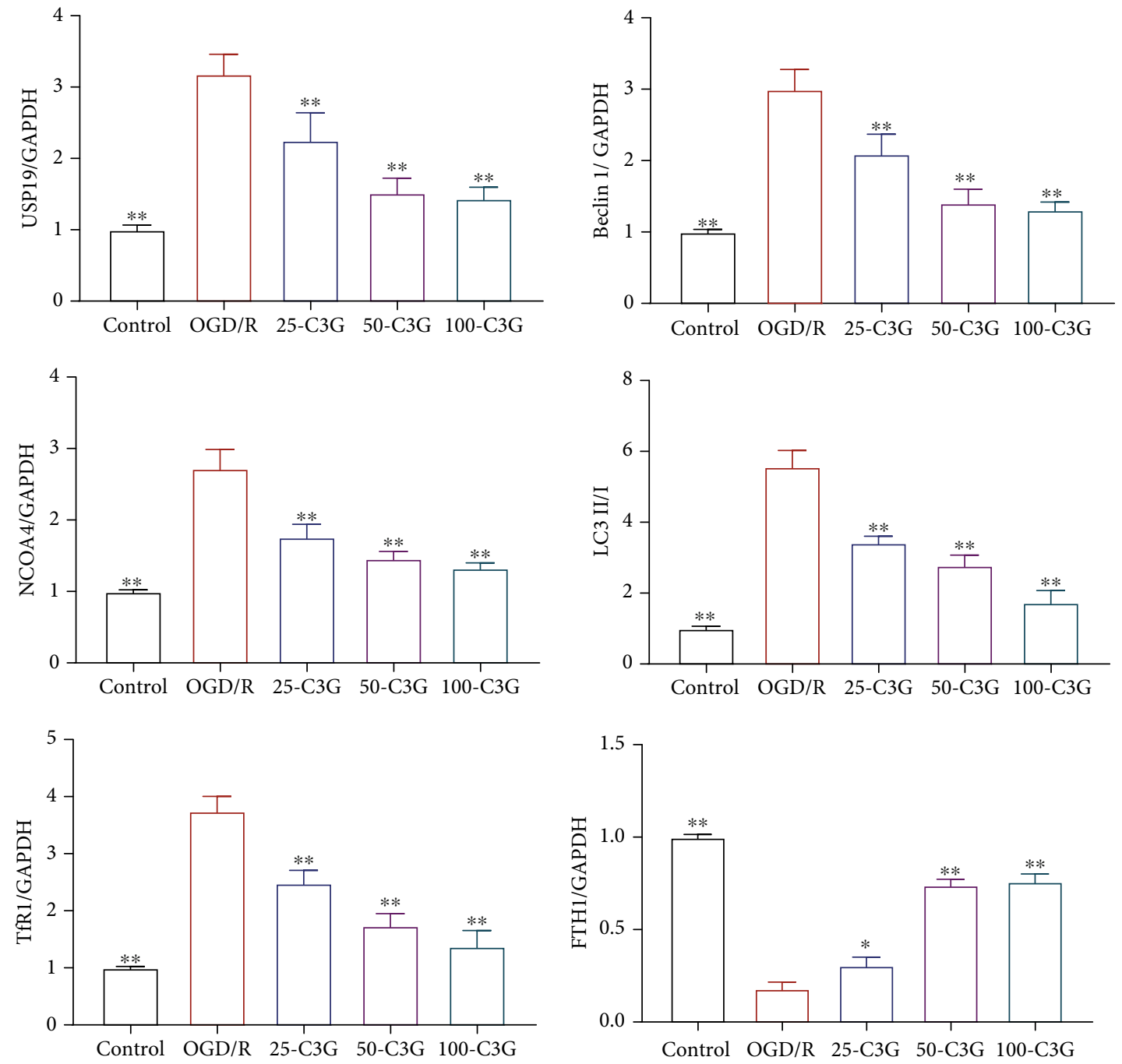

(b)

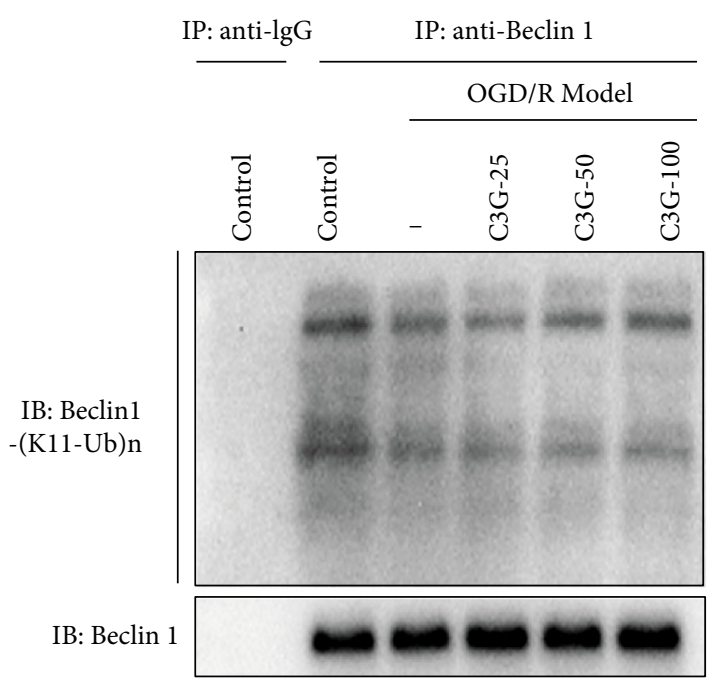

(c)

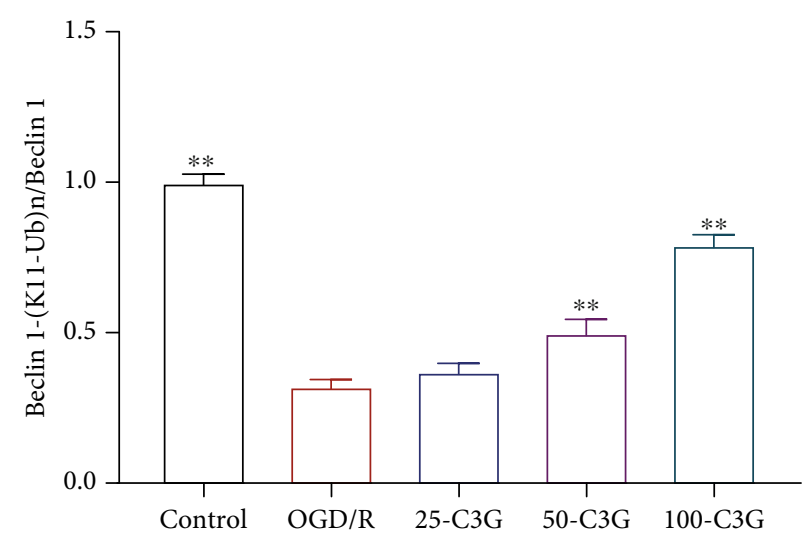

(d)

Figure 6: The effect of C3G on USP19/Beclin1-mediated ferroptosis in OGD/R-induced H9c2 cells: (a) the effect of C3G on the expressions of USP19, Beclin1, NCOA4, LC3II/LC3I, GPX4, TfR1, and FTH1; (b) the relative levels of USP19, Beclin1, NCOA4, LC3II/LC3I, GPX4, TfR1, and FTH1 versus GAPDH; (c) the effect of C3G on the K11-linked deubiquitination by co-IP; (d) the relative levels of Beclin1-(K11$\mathrm{Ub} /$ Beclin1. ${ }^{*} p<0.05,{ }^{* *} p<0.01$ compared with the OGD/R group. 


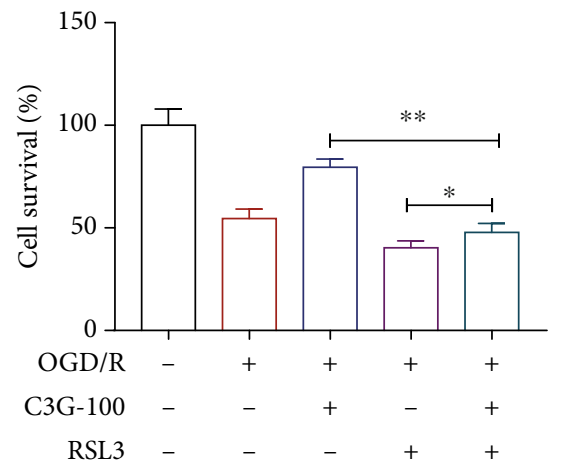

(a)
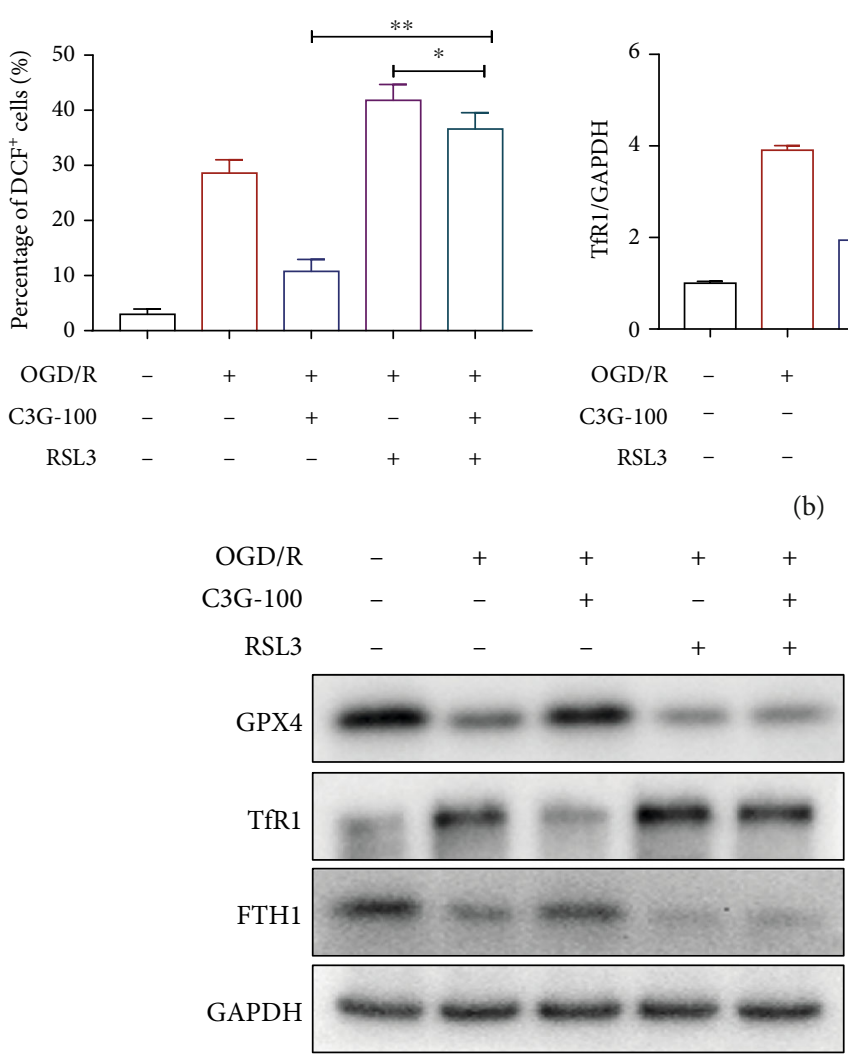

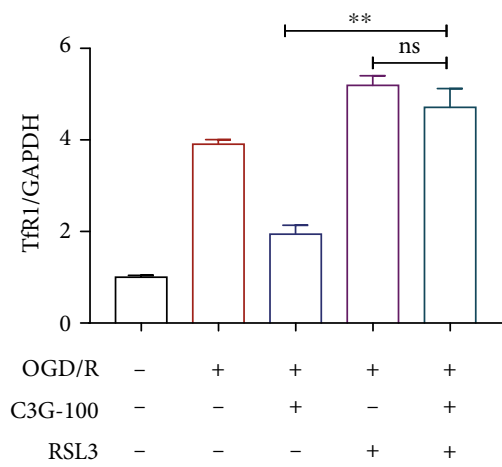

(b)

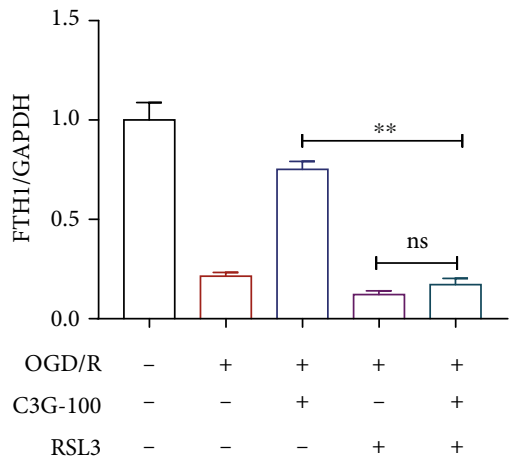

(c)

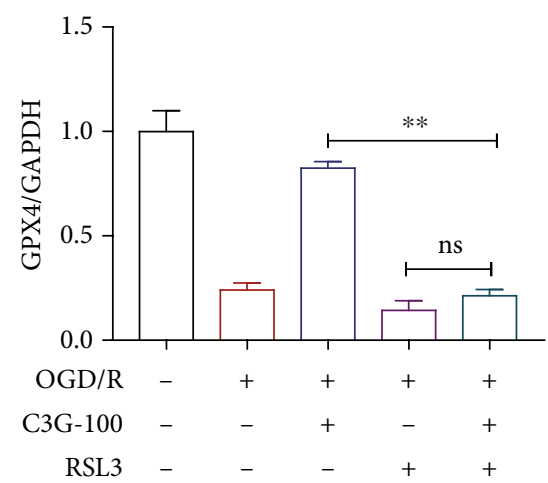

FIGURE 7: The role of C3G in RSL3-regulated autophagy. (a) C3G increased the viability of cells reduced by RSL3. (b) C3G reduced ROS levels that were increased by RSL3. (c) C3G inversely regulated the expression levels of iron death-related proteins that were altered by RSL3. When comparing the two sets of data, ${ }^{*} p<0.05$ means significant, and ns means not significant.

myocardial ischemia-reperfusion. Figure 7 (a) shows that the cell viability was significantly increased in the C3G-100 group compared to the $\mathrm{C} 3 \mathrm{G}-100+\mathrm{RSL} 3$ in the $\mathrm{OGD} / \mathrm{R}$ modeling situation and was also significantly lower in the $\mathrm{OGD} / \mathrm{R}+\mathrm{RSL} 3$ group than in the OGD/R+C3G-100 + RSL3 group $(p<0.05)$. RSL3 effectively increased the level of ROS compared to the OGD/R + RSL3 group, and treatment with $\mathrm{C} 3 \mathrm{G}$ significantly reduced the level of ROS $(p<0.05$, Figure $7(\mathrm{~b}))$. In Figure 7(c), OGD/R stimulation significantly increased the expression of TfR 1 and decreased the expression of GPX4 and FTH1, a trend that was further promoted by the addition of RSL3, indicating that RSL3 pro- moted ferroptosis. In contrast, treatment with C3G decreased the TfR1 expression and promoted the GPX4 and FTH1 expression. Notably, the use of C3G effectively blocked the promoting effect of RSL3 on ferroptosis.

\section{Discussion}

The antioxidant effects of $\mathrm{C} 3 \mathrm{G}$ can be used to treat diseases, and it was reported that $\mathrm{C} 3 \mathrm{G}$ modulated oxidation dependent on iron [14]. C3G ameliorated ferroptosis via histone ubiquitination and oxidative mitigation [15]. These literatures indicated that $\mathrm{C} 3 \mathrm{G}$ might exhibit its pharmacological 
effect through ferroptosis and ubiquitination modification. Our results demonstrated that $\mathrm{C} 3 \mathrm{G}$ administration reduced cardiac infarction area, restrained pathological damage, and inhibited ST-segment elevation, which indicated that C3G treatment effectively prevented myocardial I/R lesion.

As a degradative process crucial in cellular physiology and pathology, autophagy is activated in the pathogenesis of myocardial I/R damage. Autophagy is a catabolic process implicated with the impaired proteins and organelles, characterised primarily by the generation of autophagosomes and degradation of lysosome, which is an essential pathway to maintain cell homeostasis. Under stressful conditions of energy deficiency or hypoxia, autophagy is initiated to clear the toxic events and facilitate cell survival [16]. Autophagy accelerates availability of iron in the hypoxic environment. It is noteworthy that slight autophagy improves cell survival, while excessive autophagy is detrimental during reperfusion and is attributed to the cellular constituent consumption [17]. The upregulation of autophagy during myocardial ischemia-reperfusion may be related to the stimulus conditions (duration of ischemia). Study has reported that in mice undergoing LAD surgery, autophagic flux increased, and damaged proteins were cleared within $0-6 \mathrm{~h}$ of reperfusion, and after $6 \mathrm{~h}$, autophagic flux decreased, but the accumulation of autophagic vesicles was more pronounced, indicating that autophagy at this time was no longer able to play a role in clearing damaged proteins and was in a state of excessive autophagy to the cells [18]. The results of in vitro experiment showed that the expression of Beclin1 was upregulated in hypoxia/reoxygenation-induced $\mathrm{H} 9 \mathrm{c} 2$ cells, implying an increase in autophagy, which was consistent with previous study [19]. Results from in vitro and in vivo experiments showed that C3G suppressed the expression levels of Beclin1 and LC3II in I/R-induced cardiac tissues and OGD/Rinduced H9c2 cells and that inhibition of autophagy was benefit to the I/R model [20]. Therefore, autophagy was the key event in the ameliorated effect of $\mathrm{C} 3 \mathrm{G}$ on myocardial I/R injury.

Beclin1 activity is modulated by a variety of posttranslational modifications including ubiquitination. USP is the largest family of DUBs controlling the reversible process of ubiquitination and is a target of the unfolded protein response (UPR) [21]. Mounting evidence indicated that USP19 drives numerous cellular process. An important role for USP19 in the innate immune response has now been reported [22], with USP19 blunting pathological cardiac hypertrophy by inhibiting the inflammatory response [23]. Increasing literature indicated the correlation between USP family and autophagy. The deletion of USP14 can activate endoplasmic reticulum stress-mediated autophagy [24]. USP13 knockdown increases p-tau ubiquitination via the $20 \mathrm{~S}$ proteasome and p-tau clearance via autophagy [25]. USP8 directly deubiquitinates SQSTM1/p62 and blocks autophagy [26]. It is noteworthy that USP19 influences the ubiquitination of Beclin1, hence accelerating the production of autophagosome. USP19 stabilizes Beclin1 at residue K437 through a K11-linked polyubiquitin chains [27]. Previous investigators also confirmed that USP19 cleaves the K11-linked ubiquitin chains of Beclin1 in antivi- ral immunization and prevents its degradation via the proteasome. Our data indicated that the USP19 expression was upregulated in the I/R or OGD/R stimulation group, whereas C3G treatment downregulated the USP19 expression and blocked the K11-linked deubiquitination of Beclin1. The overexpression of USP19 by lentivirus showed that USP19 was closely associated with C3G-treated autophagy and ferroptosis.

It is acknowledged that lipid peroxidation and iron accumulation lead to ferroptosis [28]. Ferroptosis can cause cell death without the activation of caspases. Previous study has showed that expanded cardiomyopathy occurs in ferroportin-deficient cardiomyocytes [29]. As a key member of iron metabolism-associated genes, FTH1 is responsible for $\mathrm{Fe}^{2+}$ storage; TfR1 is responsible for controlling the $\mathrm{Fe}^{3+}$ transport across the cell membrane and maintaining intracellular iron homeostasis [30]. In our study, the level of ROS accumulation and iron content was increased in ex vivo models after I/R modeling. Iron overload is the key reason of myocardial cell injury [31]. Previous literature proved that the inhibition of ferroptosis exhibited a protective effect on myocardial I/R-induced rats and OGD/R H9c2 cells [32]. Our data demonstrated that $\mathrm{C} 3 \mathrm{G}$ treatment was effective in alleviating oxidative stress, reducing $\mathrm{Fe}^{2+}$ content, downregulating the TfR1 expression, and upregulating the FTH1 expression both in cells and tissues, all confirming that $\mathrm{C} 3 \mathrm{G}$ could reduce oxidative stress levels and decrease $\mathrm{Fe}^{2+}$ accumulation, both in vivo and in vitro.

\section{Conclusion}

In conclusion, our results displayed that $\mathrm{C} 3 \mathrm{G}$ attenuated myocardial I/R injury by inhibiting ferroptosis both in vivo and in vitro. The $\mathrm{C} 3 \mathrm{G}$ treatment prevented K11-linked deubiquitination of Beclin1. Further investigation with transgenic mice is warranted in the future.

\section{Abbreviations}

C3G: Cyanidin-3-glucoside

Co-IP: Coimmunoprecipitation

DHE: Dihydroethidium

DIL: Diltiazem

DUBs: Deubiquitinating enzymes

ECG: Electrocardiogram

FBS: Fetal bovine serum

FTH1: Ferritin heavy chain

IR: Ischemia-reperfusion

MOI: Multiplicity of infection

MTT: 3-(4,5-Dimethylthiazol-2-yl)-2,5diphenyltetrazoliumbromide

NCOA4: Nuclear receptor coactivator 4

OE: Overexpression

OGD/R: Oxygen-glucose deprivation/reoxygenation

ROS: $\quad$ Reactive oxygen species

SD: $\quad$ Sprague Dawley

TEM: Transmission electron microscope

TfR1: Transferrin receptor 
TTC: $\quad$ 2,3,5-Triphenyl-2H-tetrazolium chloride

UPR: Unfolded protein response

USP19: Ubiquitin-specific protease 19.

\section{Data Availability}

The data used to support the findings of this study are available from the corresponding author upon request.

\section{Conflicts of Interest}

The authors declare that they have no competing interests.

\section{Authors' Contributions}

Xin Shan and Zhiyang Lv contributed to the conceptualization and methodology, conducted the experiments, and wrote the original draft. Mengjiao Yin and Jing Chen conducted the experiments and collected the data. Jie Wang contributed to the data analysis. Qinan Wu contributed to the supervision and project administration.

\section{Acknowledgments}

The study was supported by the Chinese National Natural Science Foundation (No. 81973435), the Program of Collaborative Innovation Center of Chinese Medicinal Material Resources Industrialization of Jiangsu Province (2020), the Genuine medicinal material planting and quality assurance project (2021), the Postgraduate Research \& Practice Innovation Program of Jiangsu Province (NO. KYCX20_1496), the Jiangsu TCM science and technology development program (NO. YB201997), and the Blue Project Foundation of Jiangsu in 2018 for outstanding young backbone teachers.

\section{References}

[1] F. H. Yang, L. Xue, Z. Q. Han et al., "Vaspin alleviates myocardial ischaemia/reperfusion injury via activating autophagic flux and restoring lysosomal function," Biochemical and Biophysical Research Communications, vol. 503, no. 2, pp. 501-507, 2018.

[2] S. J. Dixon, K. M. Lemberg, M. R. Lamprecht et al., "Ferroptosis: an iron-dependent form of nonapoptotic cell death," Cell, vol. 149, no. 5, pp. 1060-1072, 2012.

[3] X. Fang, H. Wang, D. Han et al., "Ferroptosis as a target for protection against cardiomyopathy," Proceedings of the National Academy of Sciences, vol. 116, no. 7, pp. 2672-2680, 2019.

[4] K. Przyklenk, Y. Dong, V. V. Undyala, and P. Whittaker, "Autophagy as a therapeutic target for ischaemia /reperfusion injury? Concepts, controversies, and challenges," Cardiovascular Research, vol. 94, no. 2, pp. 197-205, 2012.

[5] Q. Fei, H. Ma, J. Zou et al., "Metformin protects against ischaemic myocardial injury by alleviating autophagy-ROS-NLRP3mediated inflammatory response in macrophages," Journal of Molecular and Cellular Cardiology, vol. 145, pp. 1-13, 2020.

[6] Z. Dong, S. Guo, Y. Wang et al., "USP19 enhances MMP2/MMP9-mediated tumorigenesis in gastric cancer," Oncotargets and Therapy, vol. 13, pp. 8495-8510, 2020.
[7] N. Bédard, S. Jammoul, T. Moore et al., "Inactivation of the ubiquitin-specific protease 19 deubiquitinating enzyme protects against muscle wasting," FASEB Journal, vol. 29, no. 9, pp. 3889-3898, 2015.

[8] F. J. Olivas-Aguirre, J. Rodrigo-García, N. D. Martínez-Ruiz et al., "Cyanidin-3-O-glucoside: physical-chemistry, foodomics and health effects," Molecules, vol. 21, no. 9, p. 1264, 2016.

[9] H. P. V. Rupasinghe, N. Arumuggam, M. Amararathna, and A. B. K. H. de Silva, "The potential health benefits of haskap (Lonicera caerulea L.): Role of cyanidin-3- O -glucoside," Journal of Functional Foods, vol. 44, pp. 24-39, 2018.

[10] K. Škèmienè, G. Jablonskienè, J. Liobikas, and V. Borutaite, "Protecting the heart against ischemia/reperfusion-induced necrosis and apoptosis: the effect of anthocyanins," Medicina, vol. 49, no. 2, pp. 15-88, 2013.

[11] K. Petroni, M. Trinei, M. Fornari et al., "Dietary cyanidin 3glucoside from purple corn ameliorates doxorubicin-induced cardiotoxicity in mice," Nutrition, Metabolism, and Cardiovascular Diseases: NMCD, vol. 27, no. 5, pp. 462-469, 2017.

[12] B. M. Aloud, P. Raj, J. McCallum et al., "Cyanidin 3-Oglucoside prevents the development of maladaptive cardiac hypertrophy and diastolic heart dysfunction in 20-week-old spontaneously hypertensive rats," Food \& Function, vol. 9, no. 6, pp. 3466-3480, 2018.

[13] H. C. Ku, W. P. Chen, and M. J. Su, “DPP4 deficiency preserves cardiac function via GLP-1 signaling in rats subjected to myocardial ischemia/reperfusion," Naunyn-Schmiedeberg's Archives of Pharmacology, vol. 384, no. 2, pp. 197-207, 2011.

[14] Z. Zhang, J. Li, L. Fan, and Z. Duan, "Effect of organic acid on cyanidin-3-O-glucoside oxidation mediated by iron in model Chinese bayberry wine," Food Chemistry, vol. 310, p. 125980 , 2020.

[15] X. Li, Z. Yao, D. Yang et al., "Cyanidin-3- _O_ -glucoside restores spermatogenic dysfunction in cadmium- exposed pubertal mice _via_ histone ubiquitination and mitigating oxidative damage," Journal of Hazardous Materials, vol. 387, article 121706, 2020.

[16] J. K. Salabei and D. J. Conklin, "Cardiovascular autophagy: crossroads of pathology, pharmacology and toxicology," Cardiovascular Toxicology, vol. 13, no. 3, pp. 220-229, 2013.

[17] N. Hariharan, P. Zhai, and J. Sadoshima, "Oxidative stress stimulates autophagic flux during ischemia/reperfusion," Antioxidants \& Redox Signaling, vol. 14, no. 11, pp. 2179-2190, 2011.

[18] J. Nah, P. Zhai, C. Huang et al., "Upregulation of Rubicon promotes autosis during myocardial ischemia/reperfusion injury," Journal of Clinical Investigation, vol. 130, no. 6, pp. 2978-2991, 2020.

[19] G. Guan, L. Yang, W. Huang et al., "Mechanism of interactions between endoplasmic reticulum stress and autophagy in hypoxia/reoxygenation-induced injury of H9c2 cardiomyocytes," Molecular Medicine Reports, vol. 20, no. 1, pp. 350358, 2019.

[20] Y. Zhang, D. Liu, H. Hu, P. Zhang, R. Xie, and W. Cui, "HIF-1 $\alpha /$ BNIP3 signaling pathway-induced-autophagy plays protective role during myocardial ischemia-reperfusion injury," Biomedicine \& Pharmacotherapy, vol. 120, p. 109464, 2019.

[21] G. C. Hassink, B. Zhao, R. Sompallae et al., “The ER-resident ubiquitin-specific protease 19 participates in the UPR and 
rescues ERAD substrates," EMBO Reports, vol. 10, no. 7, pp. 755-761, 2009.

[22] X. Wu, C. Lei, T. Xia, X. Zhong, Q. Yang, and H. B. Shu, "Regulation of TRIF-mediated innate immune response by K27-linked polyubiquitination and deubiquitination," Nature Communications, vol. 10, no. 1, p. 4115, 2019.

[23] R. Miao, Y. Lu, X. He, X. Liu, Z. Chen, and J. Wang, "Ubiquitin-specific protease 19 blunts pathological cardiac hypertrophy via inhibition of the TAK1-dependent pathway," Journal of Cellular and Molecular Medicine, vol. 24, no. 18, pp. 10946-10957, 2020.

[24] A. A. Moghadami, E. Aboutalebi Vand Beilankouhi, A. Kalantary-Charvadeh et al., "Inhibition of USP14 induces ER stress-mediated autophagy without apoptosis in lung cancer cell line A549," Cell Stress and Chaperones, vol. 25, no. 6, pp. 909-917, 2020.

[25] X. Liu, M. L. Hebron, S. Mulki et al., "Ubiquitin specific protease 13 regulates Tau accumulation and clearance in models of Alzheimer's disease," Journal of Alzheimer's Disease, vol. 72, no. 2, pp. 425-441, 2019.

[26] H. Peng, F. Yang, Q. Hu et al., “The ubiquitin-specific protease USP8 directly deubiquitinates SQSTM1/p62 to suppress its autophagic activity," Autophagy, vol. 16, no. 4, pp. 698-708, 2020.

[27] J. Cui, S. Jin, and R. F. Wang, "The BECN1-USP19 axis plays a role in the crosstalk between autophagy and antiviral immune responses," Autophagy, vol. 12, no. 7, pp. 1210-1211, 2016.

[28] J. P. F. Angeli, R. Shah, D. A. Pratt, and M. Conrad, "Ferroptosis inhibition: mechanisms and opportunities," Trends in Pharmacological Sciences, vol. 38, no. 5, pp. 489-498, 2017.

[29] S. Lakhal-Littleton, M. Wolna, C. A. Carr et al., "Cardiac ferroportin regulates cellular iron homeostasis and is important for cardiac function," Proceedings of the National Academy of Sciences, vol. 112, no. 10, pp. 3164-3169, 2015.

[30] J. Y. Cao and S. J. Dixon, "Mechanisms of ferroptosis," Cellular and Molecular Life Sciences, vol. 73, no. 11-12, pp. 2195-2209, 2016.

[31] E. Park and S. W. Chung, "ROS-mediated autophagy increases intracellular iron levels and ferroptosis by ferritin and transferrin receptor regulation," Cell Death \& Disease, vol. 10, no. 11, p. 822, 2019.

[32] W. Li, W. Li, Y. Leng, Y. Xiong, and Z. Xia, "Ferroptosis is involved in diabetes myocardial ischemia/reperfusion injury through endoplasmic reticulum stress," DNA and Cell Biology, vol. 39, no. 2, pp. 210-225, 2020. 